\title{
Distribution of agricultural pesticides in the freshwater environment of the Guayas river basin (Ecuador)
}

Arne Deknock, Niels De Troyer, Michael Houbraken, Luis Dominguez-Granda, Indira Nolivos, Wout Van Echelpoel, Marie Anne Eurie Forio, Pieter Spanoghe, Peter Goethals

2 October 2019

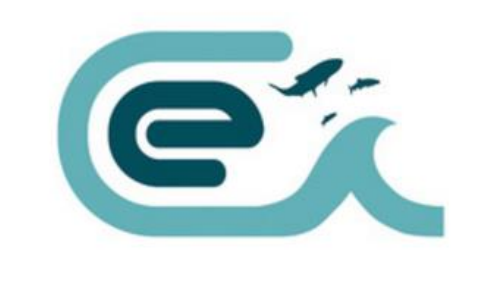




\section{INTRODUCTION}

- Majority of applied pesticides ends up in environment

- Biologically active

- Developing regions:

- Non-specific application methods
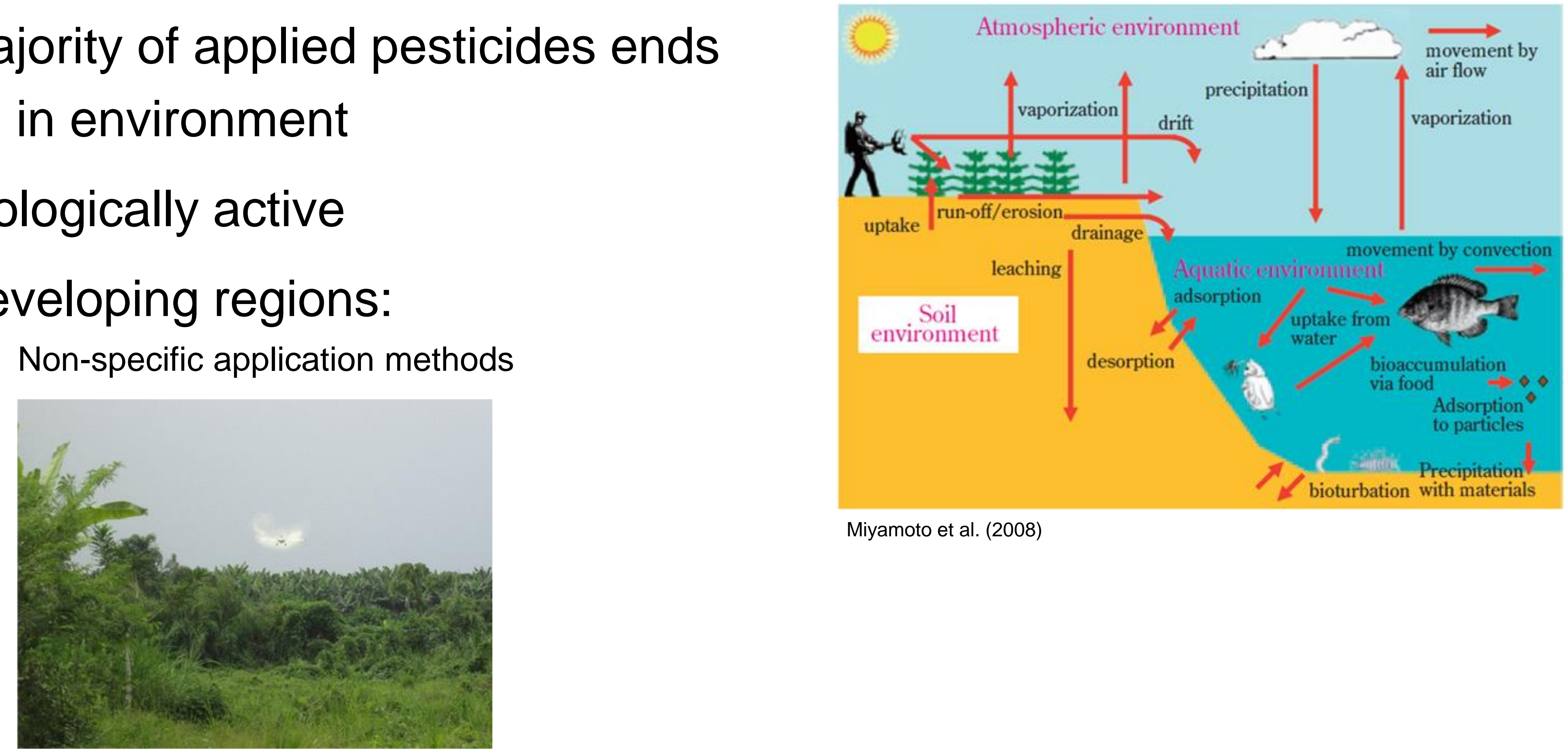

Miyamoto et al. (2008) 


\section{INTRODUCTION}

- Majority of applied pesticides ends up in environment

- Biologically active

- Developing regions:

- Non-specific application methods

- Cheap products (e.g. persistent organochlorines)

- Lack of training, technical services, regulations and control

- Low awareness and integration of rational pest management strategies

- Pressure by multinationals

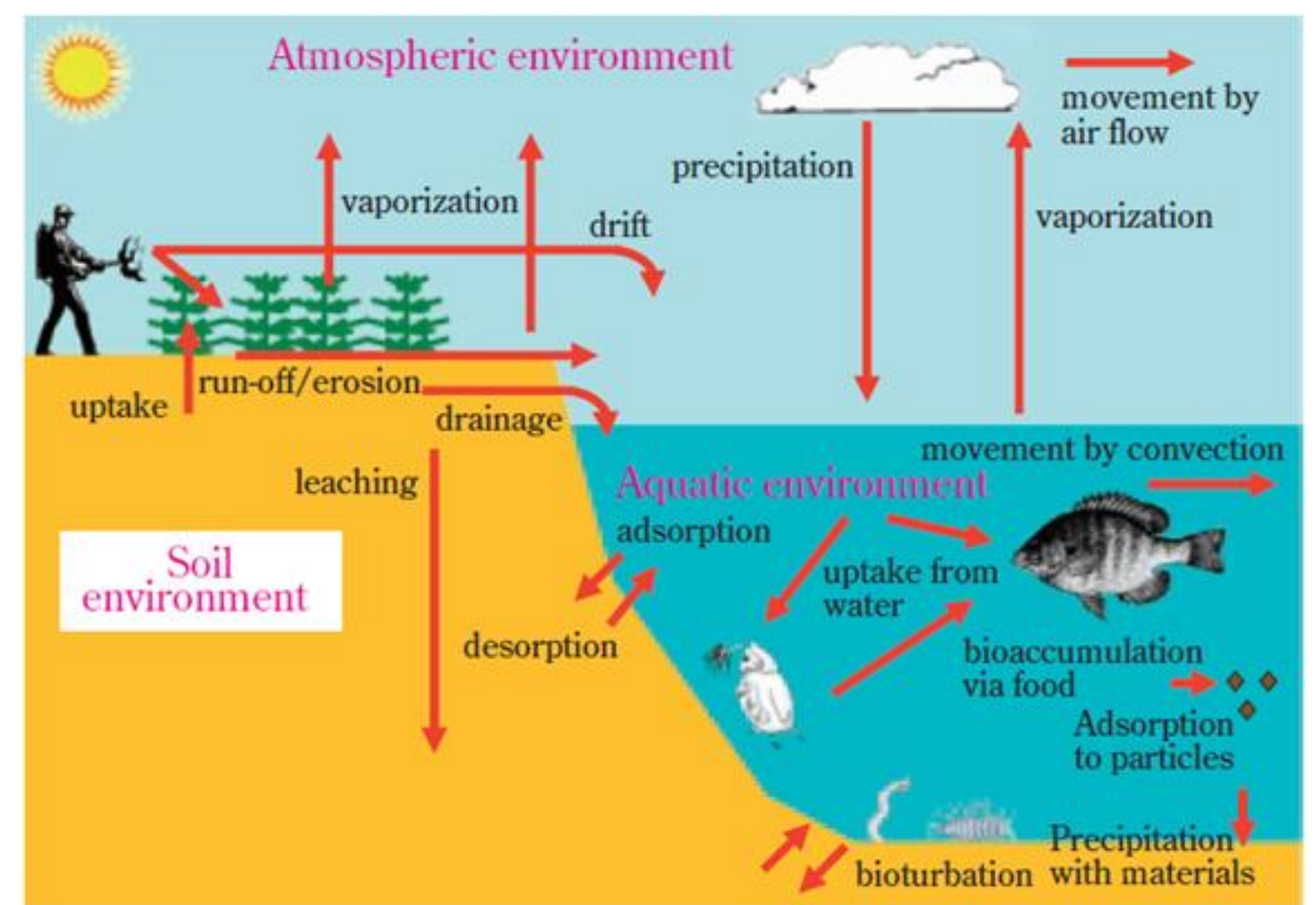

Miyamoto et al. (2008) 


\section{INTRODUCTION}

- Majority of applied pesticides ends up in environment

- Biologically active

- Developing regions:

$$
\text { Q SEARCH LAW.COM }
$$

Chiquita Can't Escape Ecuadorean Farm Workers' Lawsuit Over Pesticide Poisoning

A Delaware federal magistrate judge has denied produce company Chiquita's request to be let out of Ilitigation concerning

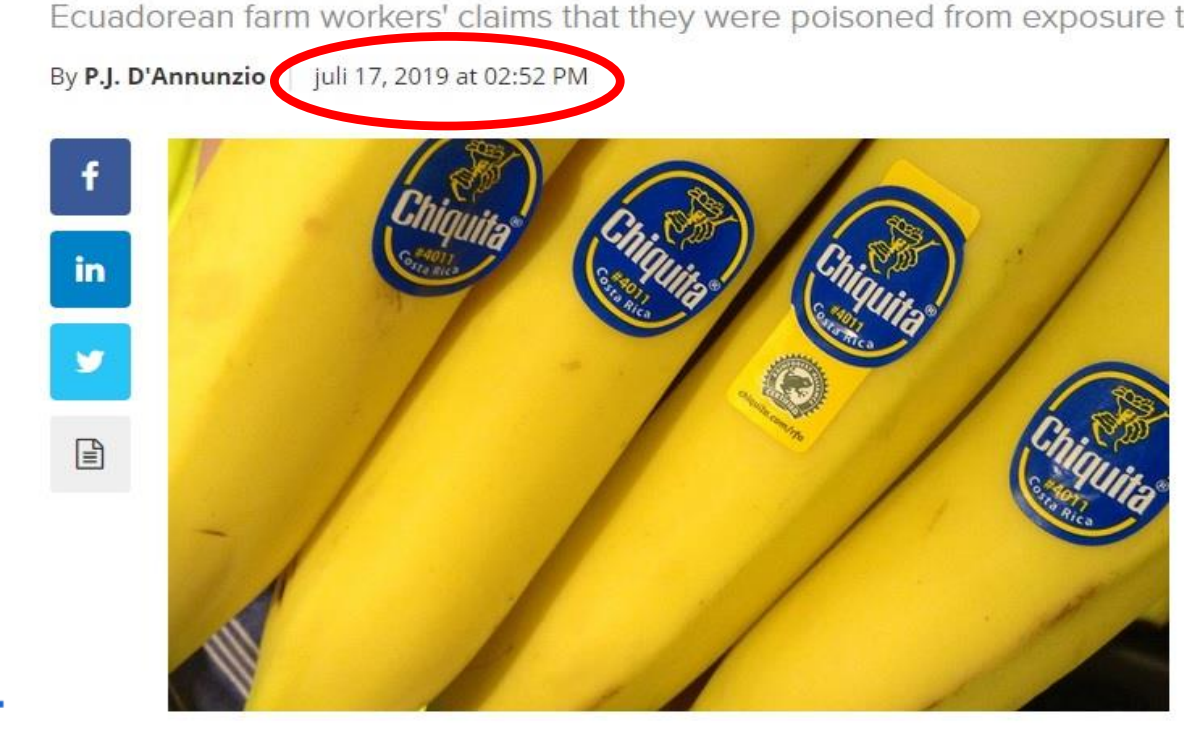

Law Firms Mentioned
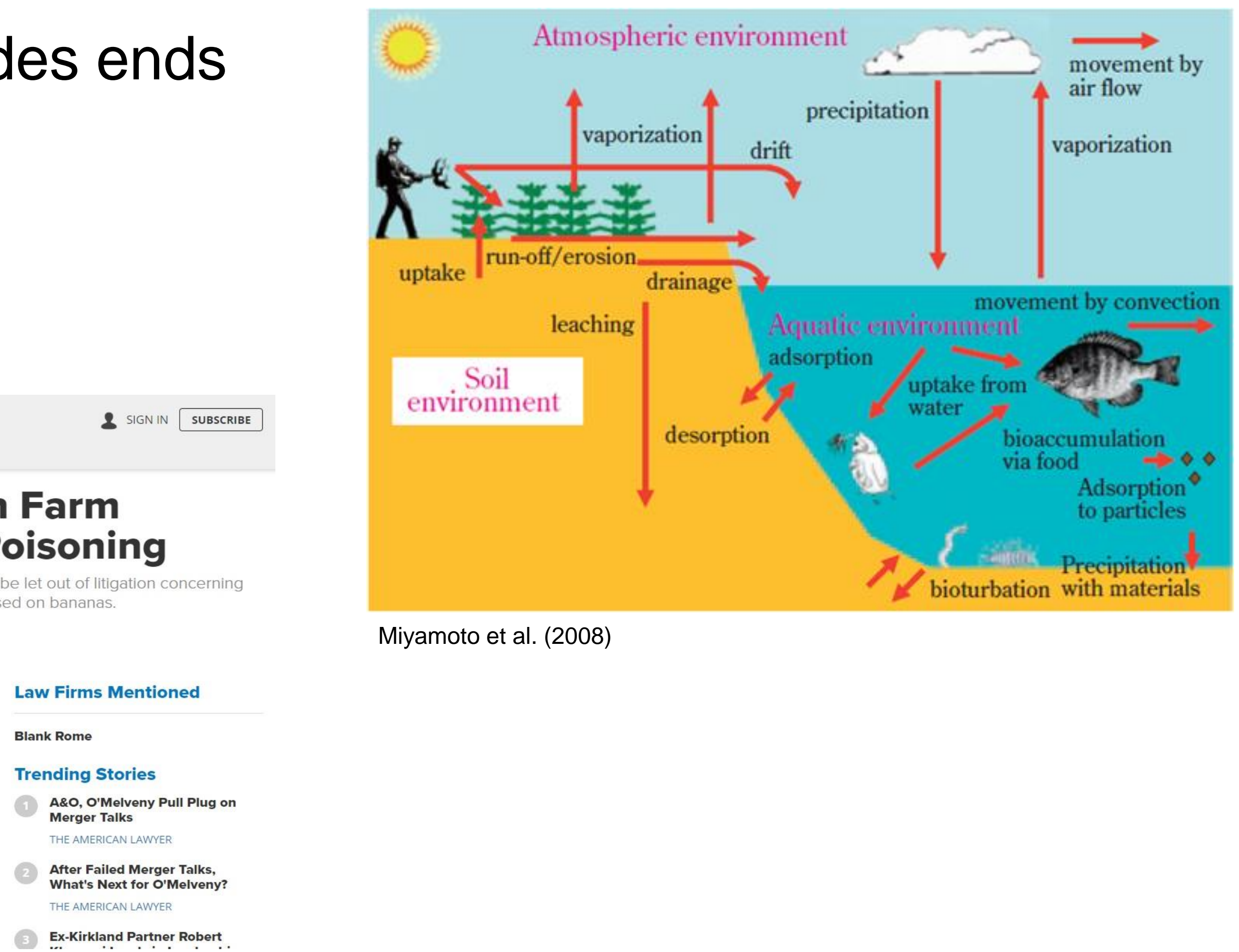

Miyamoto et al. (2008) 


\section{INTRODUCTION}

\section{- Ecuador}

- largest export-based banana industry

- 34,317 metric tons of active ingredients per year (2014-2017)

- Guayas river basin

- $40 \%$ of national population (2002)

- $68 \%$ of national crop production (1996)

- $88 \%$ of Ecuadorean bananas (1996)

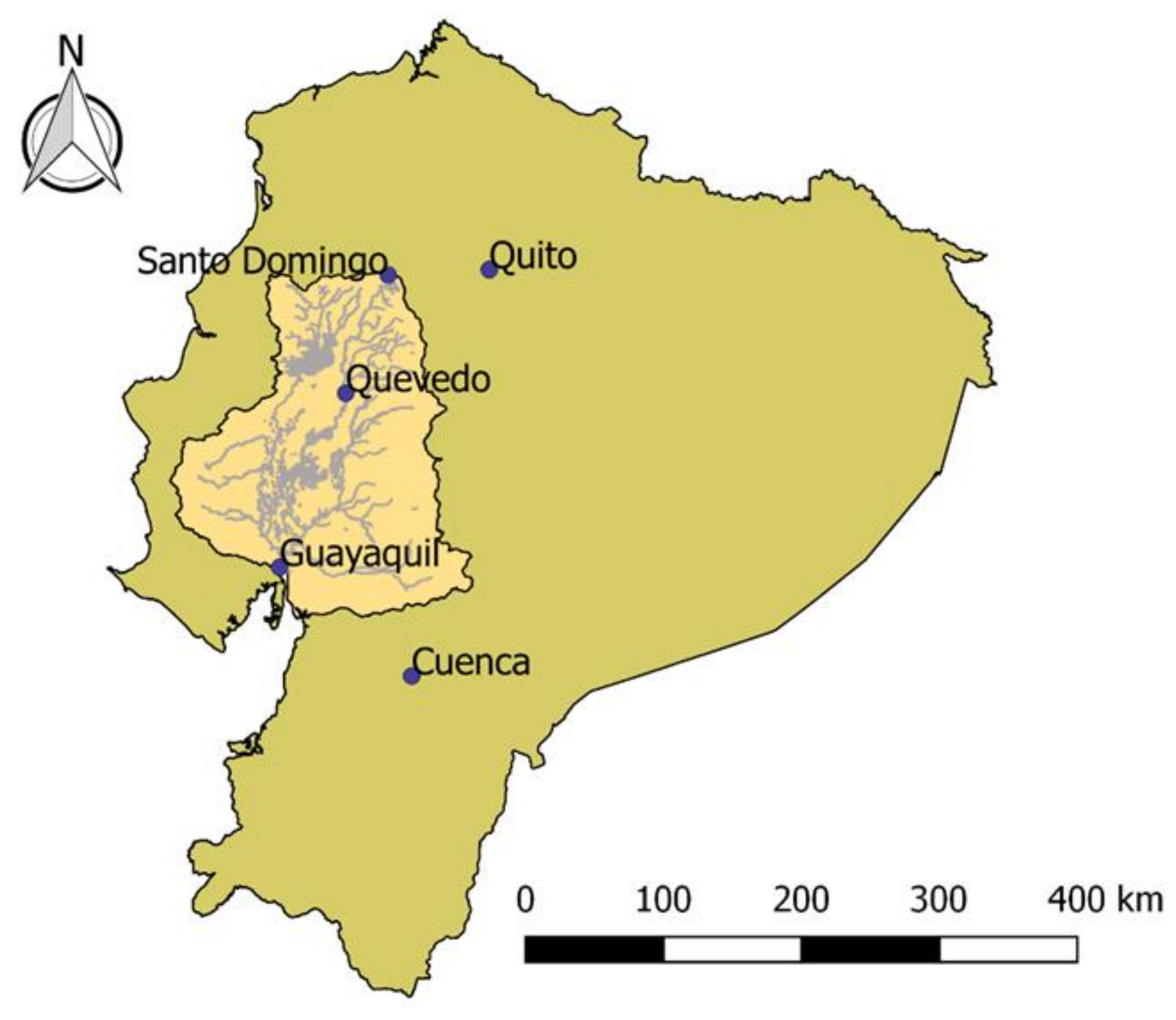




\section{OBJECTIVES}

1. Pesticide residues within the Guayas river basin?

2. Link with agricultural land use? 


\section{METHODOLOGY}

- July and August 2016

- 181 sampling locations

- 31 Daule-Peripa reservoir

- 15 Manglares Churute natural reserve

- Including upstream, downstream, natural and clearly affected locations

- Selection of 83 relevant products

- Solid-phase extraction (SPE)

$$
\text { Sep-Pak }
$$

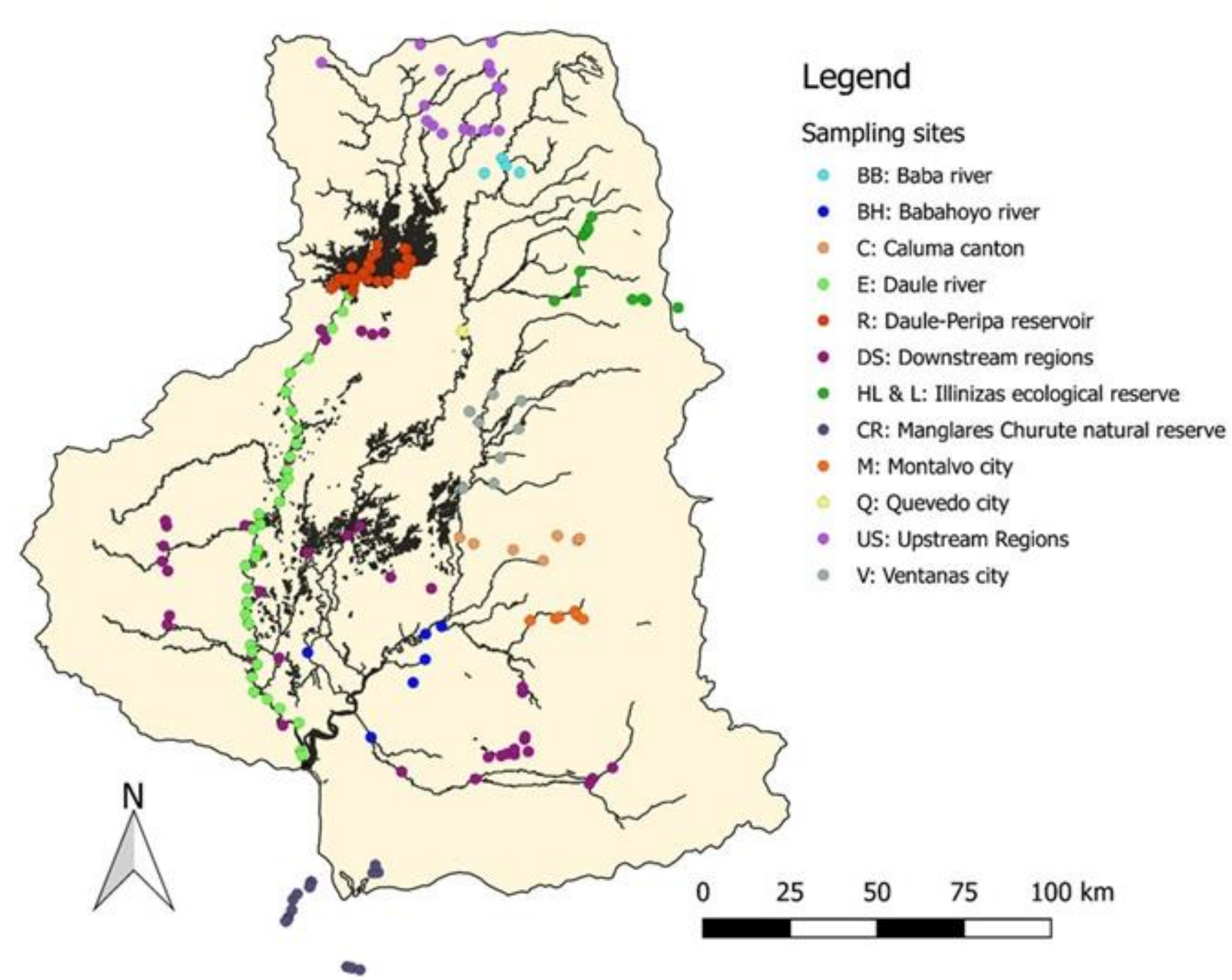




\section{METHODOLOGY}

- Gas chromatography with electron capture detector (GC-ECD) and liquid chromatography tandem-mass spectrometry (LC-MS/MS)
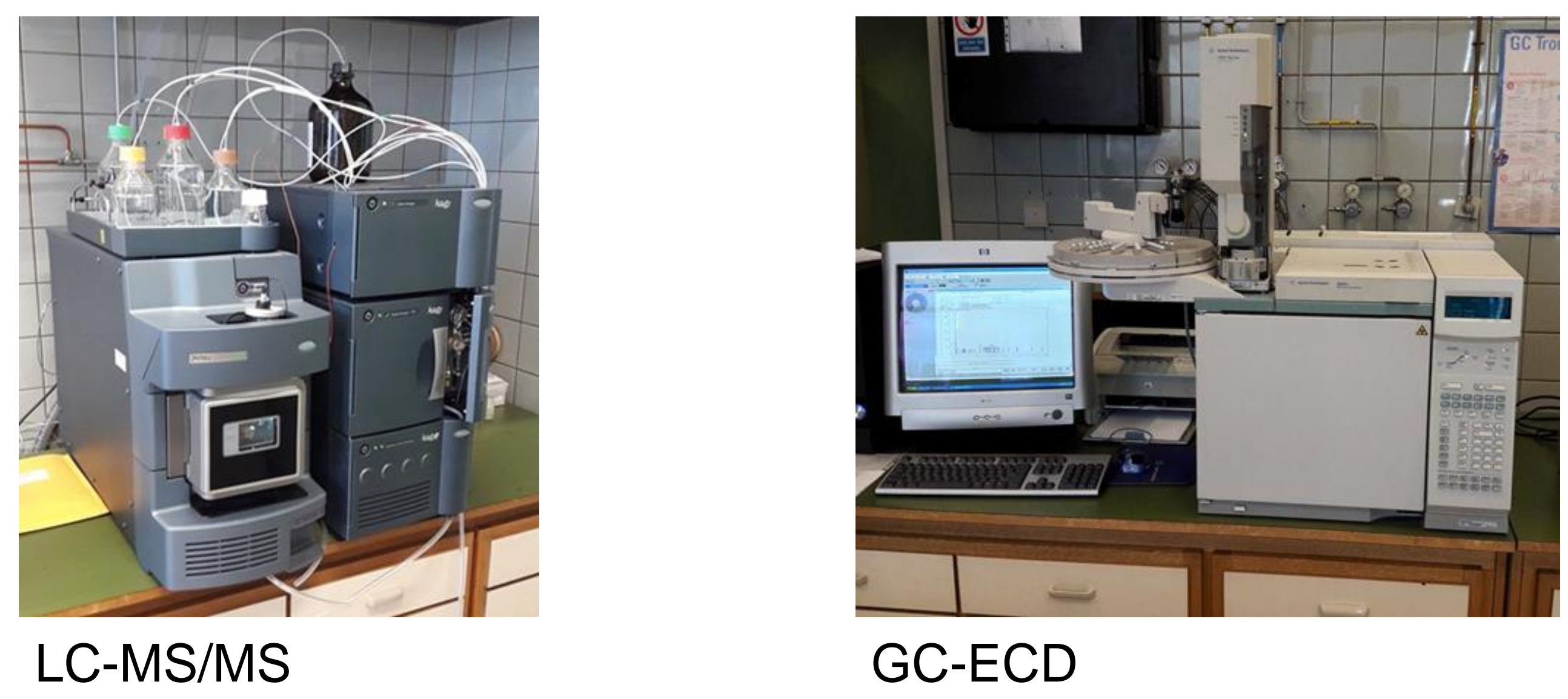

- Validation: spike-placebo recovery method 


\section{OBJECTIVES}

1. Pesticide residues within the Guayas river basin?

2. Link with agricultural land use? 
- 26 products

- 108 contaminated sites (60\%)

- Highest product diversity:

- Daule river (E-series): 8 products

- Babahoyo river (BH-series): 7 products

- Downstream region more polluted

- No impact on biological diversity

- Note:

- Single time point

- Other compartments?

\begin{tabular}{llll}
\hline Pesticide & \# locations & Concentration range $\left(\mu \mathrm{g} \cdot \mathrm{L}^{-1}\right)$ & Recovery $(\%)$ \\
\hline Acephate & 3 & $<\mathrm{LOQ}$ & $58.21 \pm 1.60$ \\
Ametryn & 1 & $<\mathrm{LOQ}$ & $81.49 \pm 5.54$ \\
Boscalid & 2 & $<\mathrm{LOQ}$ & $97.59 \pm 4.88$ \\
Butachlor & 21 & $<\mathrm{LOQ}-2.006$ & $78.10 \pm 21.77$ \\
Cadusafos & 62 & $<\mathrm{LOQ}-0.081$ & $61.73 \pm 6.12$ \\
Chlorpyrifos & 5 & $<\mathrm{LOQ}-0.035$ & $62.70 \pm 9.16$ \\
Diazinon & 3 & $<\mathrm{LOQ}$ & $63.10 \pm 28.24$ \\
Fenpropimorph & 15 & $0.022-0.241$ & $98.27 \pm 22.26$ \\
Linuron & 6 & $<\mathrm{LOQ}$ & $98.50 \pm 30.85$ \\
Malathion & 12 & $<\mathrm{LOQ}-0.687$ & $119.83 \pm 6.60$ \\
Metalaxyl & 3 & $<\mathrm{LOQ}$ & $99.75 \pm 6.87$ \\
Oxadiazon & 2 & $0.068-0.120$ & $108.80 \pm 27.35$ \\
Parathion & 1 & $<\mathrm{LOQ}$ & $105.03 \pm 6.48$ \\
Pendimethalin & 21 & $0.170-0.557$ & $94.97 \pm 11.40$ \\
Pyraclostrobin & 1 & $<\mathrm{LOQ}$ & $44.31 \pm 13.75$ \\
Pyrimethanil & 11 & $<\mathrm{LOQ}-0.080$ & $47.92 \pm 6.41$ \\
Spiroxamine & 1 & 0.099 & $82.85 \pm 7.30$ \\
Tebuconazole & 1 & 0.316 & $61.34 \pm 2.94$ \\
Terbutryn & 1 & $<\mathrm{LOQ}$ & $76.78 \pm 6.32$ \\
Triadimenol & 1 & 0.092 & $64.50 \pm 13.83$ \\
\hline
\end{tabular}

${ }^{\mathrm{a}} \mathrm{LOQ}=0.022 \mu \mathrm{g} \cdot \mathrm{L}^{-1}$ for all mentioned pesticides. 


\section{OBJECTIVES}

1. Pesticide residues within the Guayas river basin?

2. Link with agricultural land use? 


\section{RESULTS \& DISCUSSION}

\section{Cadusafos (62 sites)}

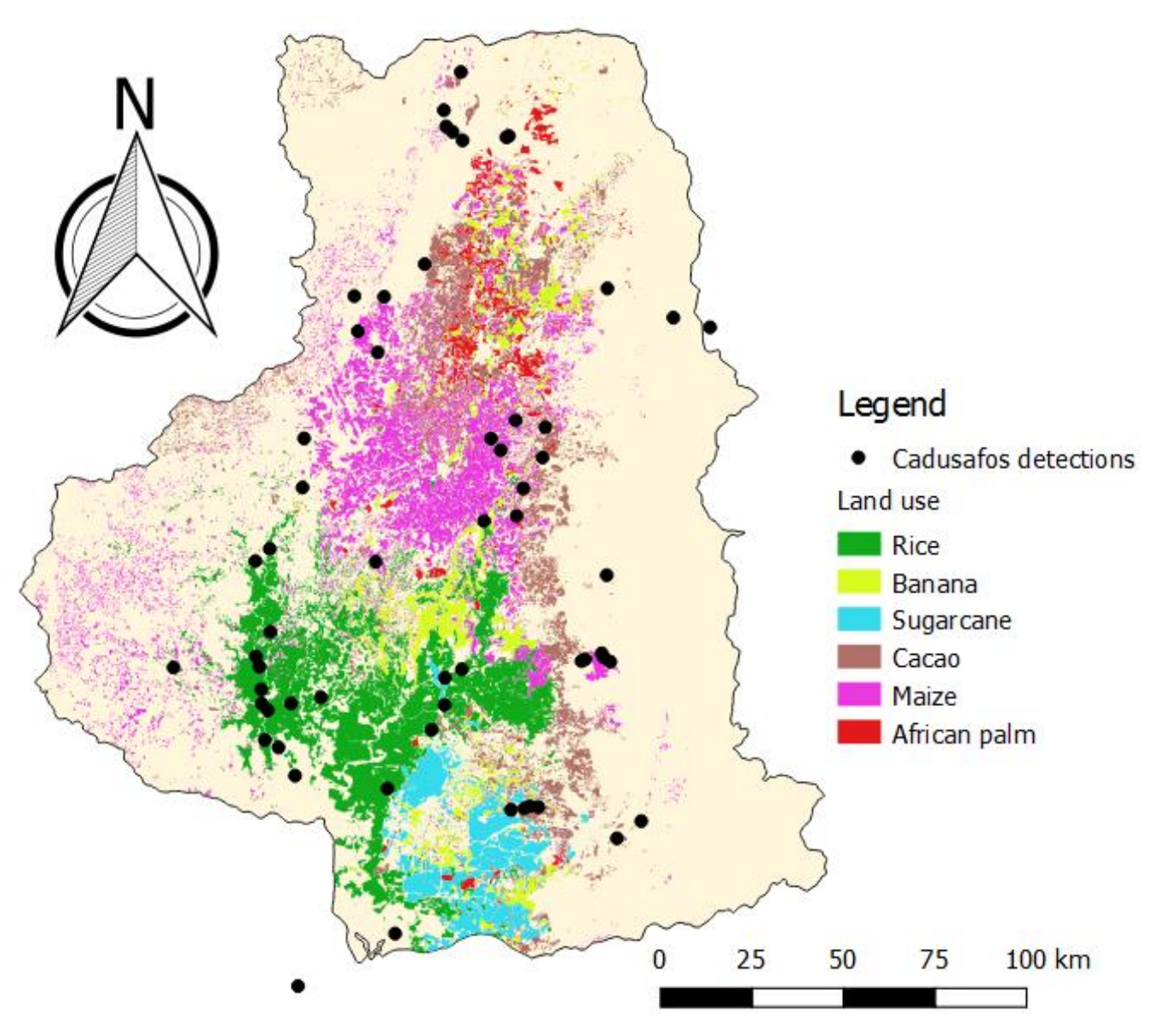

- Widely spread

- Frequently used insecticide in banana industry

- Slow degradation: DT50 $=215 \mathrm{~d}$

$\widehat{\widehat{N I I I}} \cdot$ Relatively mobile: $\mathrm{S}=245 \mathrm{mg} / \mathrm{L}$, GHENT

\section{Butachlor (21 sites)}
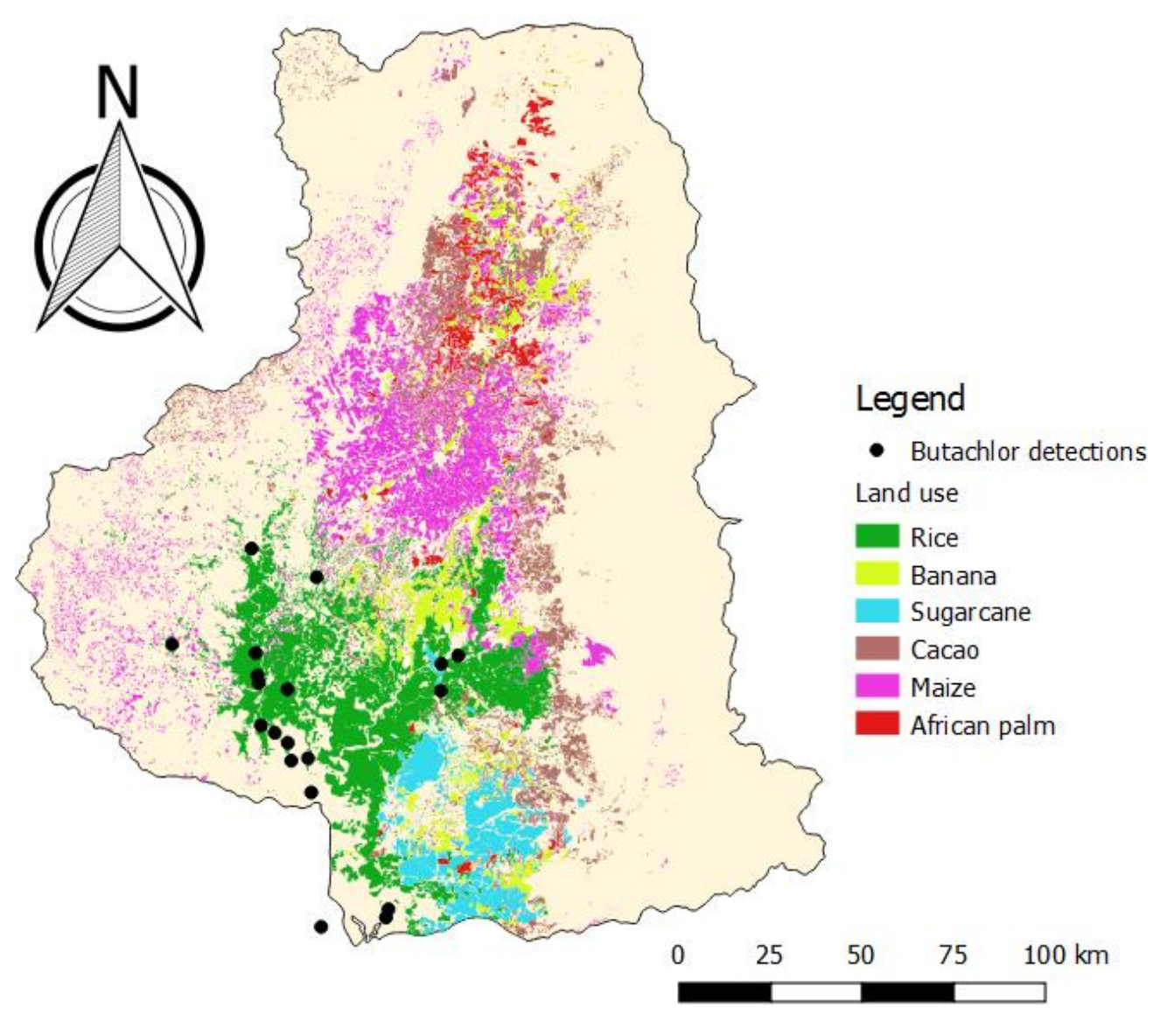

- Concentrated in rice field areas

- Commonly used herbicide in cereal cultivation to control grassy weeds

- Very immobile: $\mathrm{S}=20 \mathrm{mg} / \mathrm{L}$, $\mathrm{Koc}=700 \mathrm{~L} / \mathrm{kg}$

\section{Pendimethalin (21 sites)}

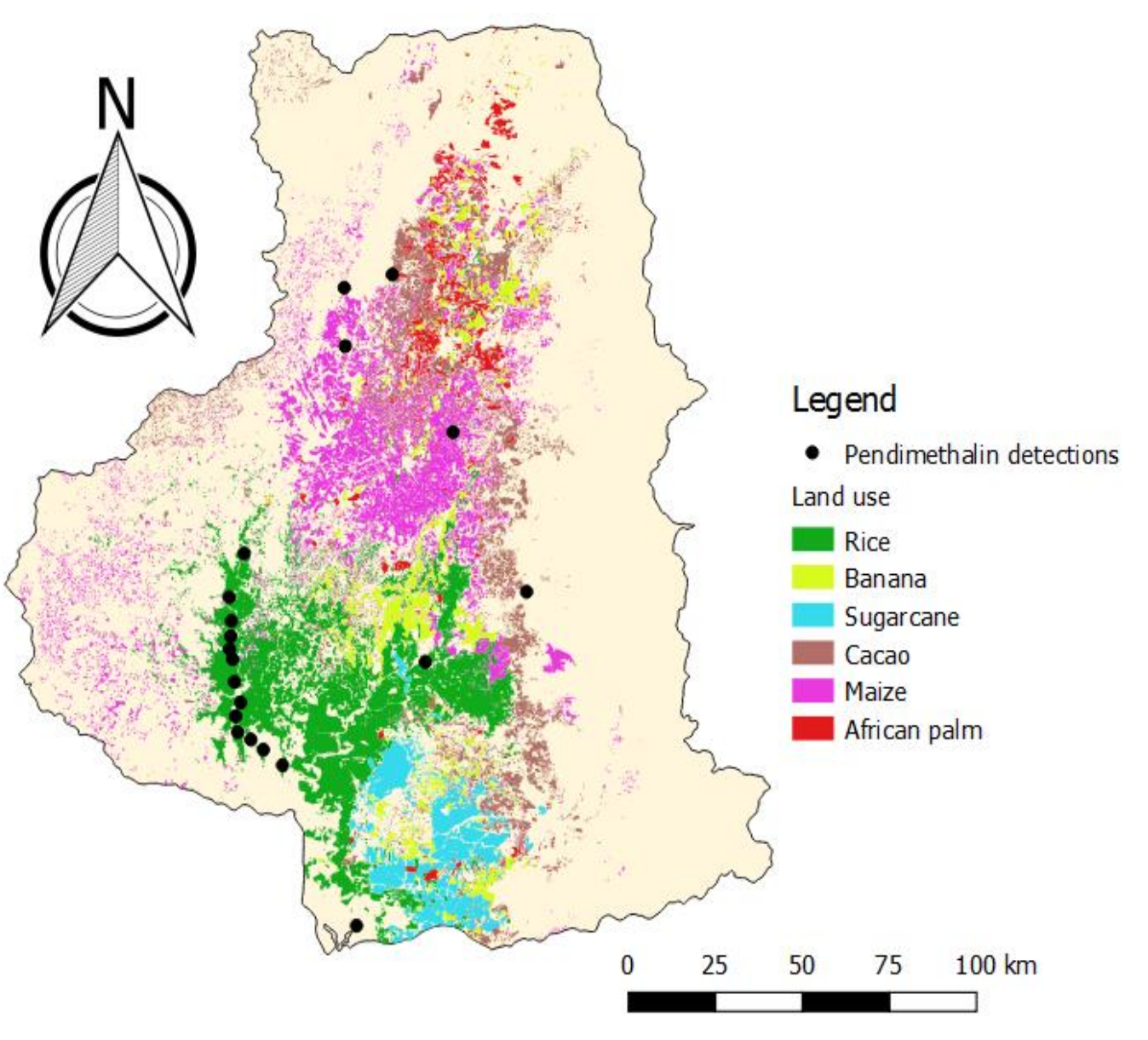

- Daule river

- Mainly detections in rice field areas, and few near maize and cacao

- Commonly used herbicide in cereal cultivation to control grassy weeds

- Very immobile: $\mathrm{S}=0.33 \mathrm{mg} / \mathrm{L}$, $\mathrm{Koc}=17,491 \mathrm{~L} / \mathrm{kg}$ 


\section{RESULTS \& DISCUSSION}

\section{Fenpropimorph (15 sites)}

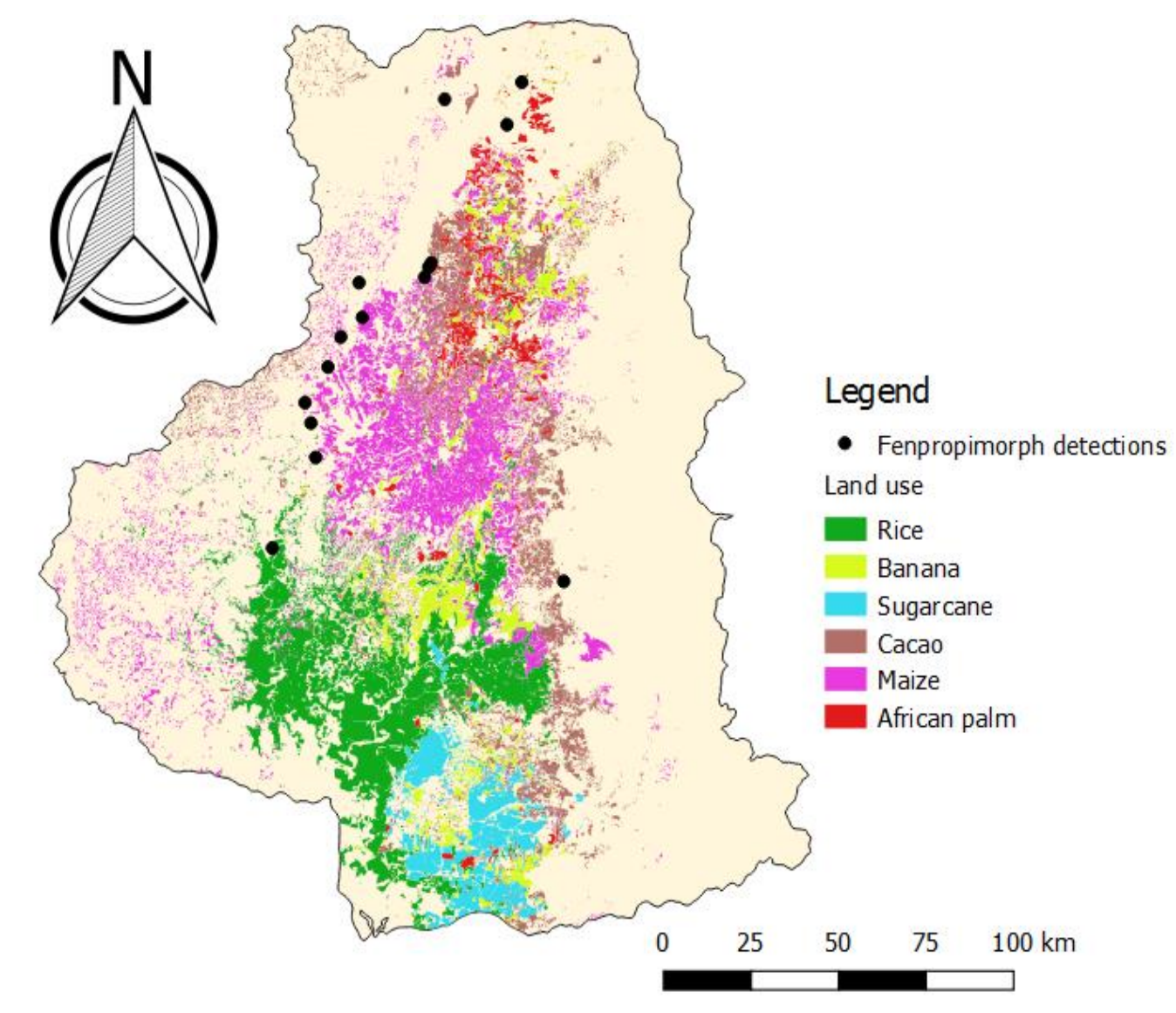

- Upstream region near maize and cacao, infiltrated with banana plantations

- Frequently used fungicide in cereal

$\begin{array}{ll}\widehat{\widehat{\text { IIIIIII }}} & \begin{array}{l}\text { and banana industries } \\ \text { GHENT }\end{array} \\ \text { No physicochemical data }\end{array}$

\section{Malathion (12 sites)}

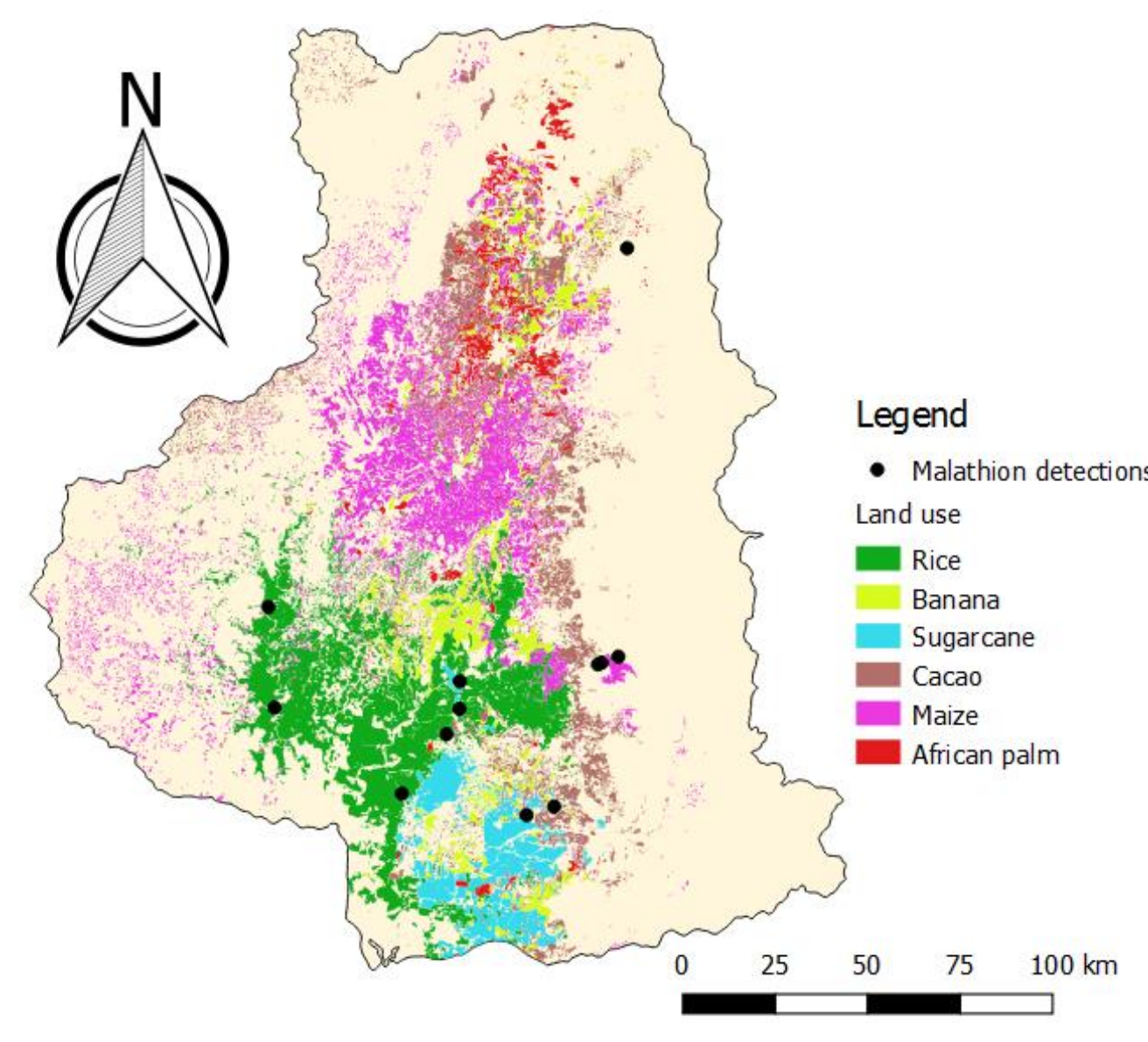

- Downstream region (Babahoyo river)

- Broad-spectrum insecticide

- Very fast degradation: DT50 = $0.4 \mathrm{~d}$

- Slightly mobile: $\mathrm{S}=148 \mathrm{mg} / \mathrm{L}$, $\mathrm{Koc}=1,800 \mathrm{~L} / \mathrm{kg}$

\section{Pyrimethanil (11 sites)}

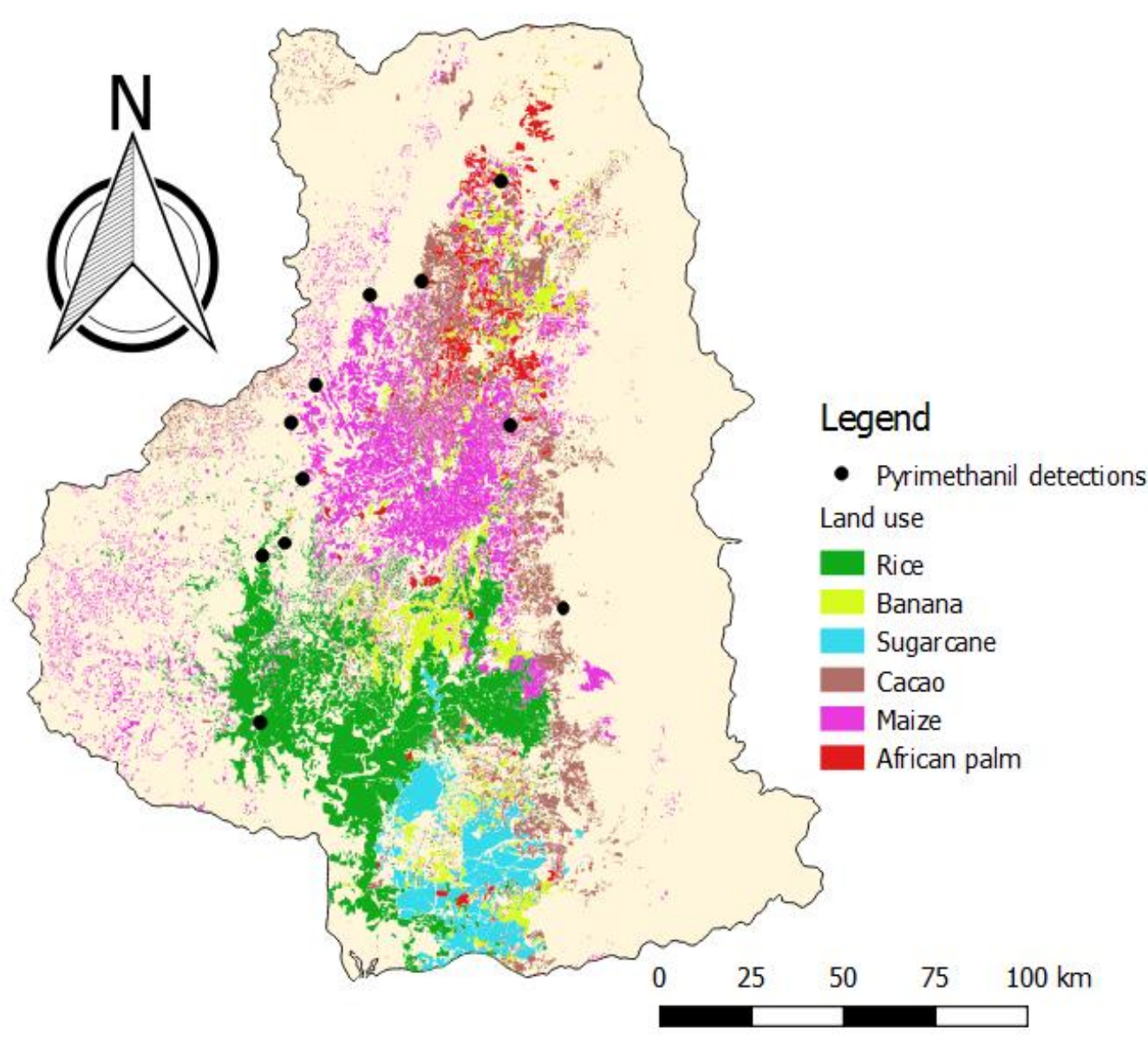

- Widely spread

- Broad-spectrum fungicide

- No physicochemical data 


\section{RESULTS \& DISCUSSION}

- Pesticide residues $\Leftrightarrow$ agricultural land use

- Banana and rice

- High consumption rates

- Application methods

- Alternatives and solutions?

- Ground-based spraying systems

- Avoid monocultures (agroforestry)

- Integrated Pest Management strategy (IPM)

- Legal regulations + control

- Awareness campaigns + training

- Policy changes multinationals
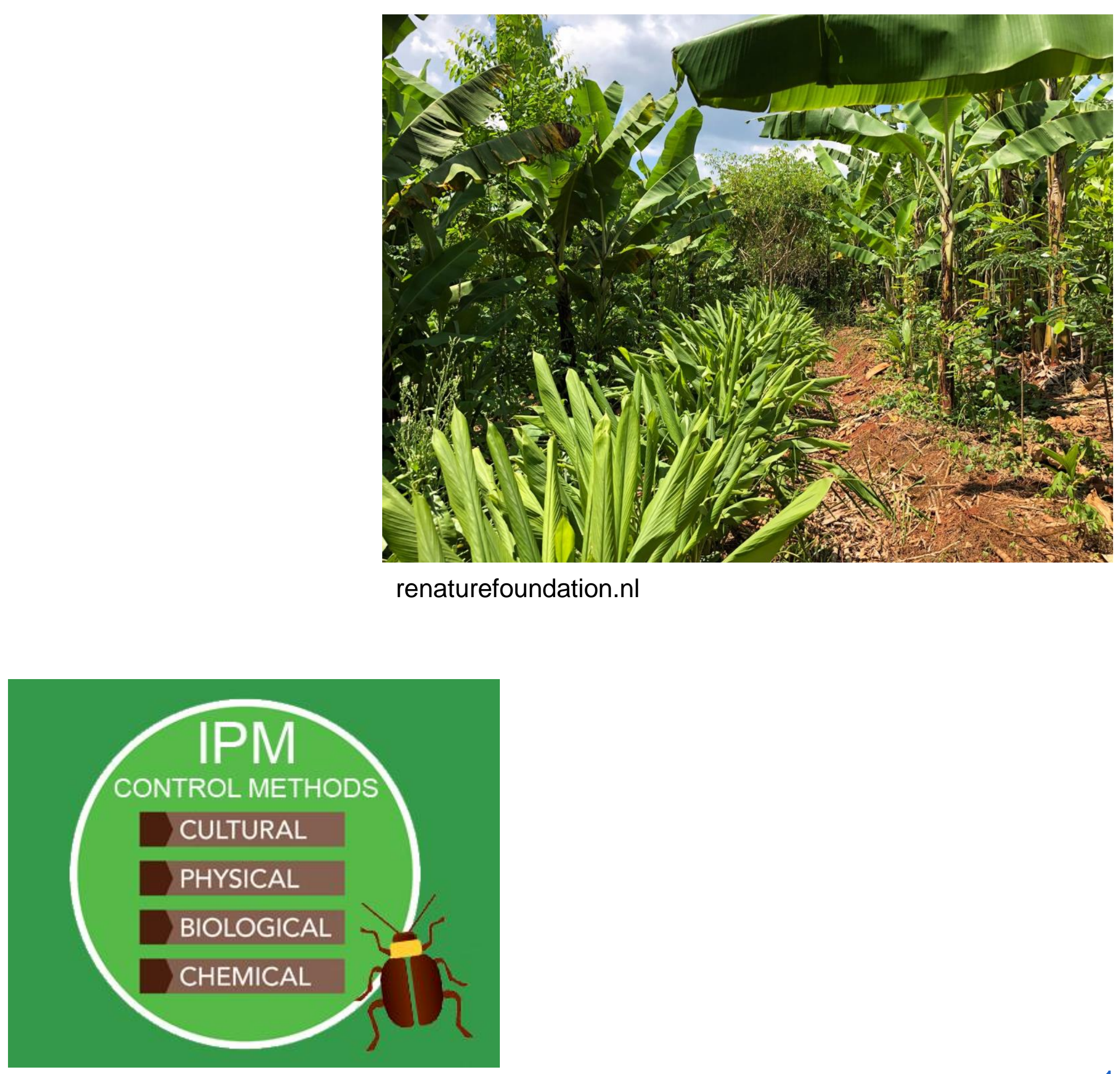

ecpa.eu 


\section{CONCLUSIONS}

- Pesticide residues widely present in Guayas river basin

- No impact on biological diversity

- Only water phase: other compartments?

- Single time point: rainy season? Production cycles?

- Frequently detected (>10 sites) were linked with agricultural land use

- Banana and rice

- High consumption rates + application methods

- Alternatives: changing cultivation methods, awareness campaigns, training programs, regulatory measures, policy changes from multinationals 


\section{Arne Deknock}

Doctoral student

AQUATIC ECOLOGY (AECO)

f Universiteit Gent

У @ugent

arne.deknock@ugent.be

www.ugent.be

우 @ugent

in Ghent University 